7. Appell has shown that the system of trajectories of a particle moving in a (positional) field of force is converted by any projective transformation into the system of trajectories corresponding to some other field of force. Professor Kasner showed, synthetically and analytically, that no other contact transformations possess this property.

8. Professor Miller's first paper appears in full in the present number of the BulLETIN.

9. Professor Miller's second paper is devoted to an extension of the results in the paper on "Non-abelian groups in which every subgroup is abelian" published in volume 4 of the Traneactions. The principal theorems may be stated as follows: There is one and only one group of order $2^{m}$ which involves operators whose orders exceed four and satisfies the additional conditions that every subgroup is either abelian or hamiltonian and that at least one subgroup is hamiltonian. If every subgroup of a group of order $2^{m}, m>4$, is either abelian or hamiltonian and if it contains at least one hamiltonian subgroup, the entire group is hamiltonian. If a group contains at least one hamiltonian subgroup and if all its subgroups are either abelian or hamiltonian, it is the direct product of the hamiltonian group of order $2^{m}$ and an abelian group of odd order, unless it is the group of order 24 which does not contain a subgroup of order 12. There are only two non-hamiltonian groups which contain at least one hamiltonian subgroup and whose other subgroups are either abelian or hamiltonian. Secretary.

\title{
THE STUTTGART MEETING OF THE DEUTSCHE MATHEMATIKER-VEREINIGUNG.
}

The annual meeting of the Deutsche Mathematiker-Vereinigung was held at Stuttgart September 17-20, 1906, forming a section of the seventy-eighth convention of the Deutsche Naturforscher und Aerzte. The following papers were presented :

(1) O. Blumenthal, Aachen : "Integral transcendental functions and Picard's theorem " (report).

(2) A. Pringsheim, Munich : "Fourier's integral theorem." 
(3) G. Faber, Karlsruhe: "Series of Legendre's polynomials."

(4) O. Perron, Munich : "Singular points on the circle of convergence."

(5) F. Hartogs, Munich : "Recent investigations of analytic functions of several variables" (report).

(6) P. Stäckel, Hanover : "Power series in several variables."

(7) D. HilberT, Göttingen : "The nature and purposes of the theory of integral equations."

(8) E. Hilb, Erlangen : “ An extension of Klein's oscillation theorem."

(9) M. Krause, Dresden : "On functions of real variables."

(10) P. Koebe, Göttingen : "On the conformal mapping of multiply connected plane regions."

(11) F. Meyer, Koenigsberg: "The application of the generalized euclidean algorithm to the formation of resultants."

(12) P. Schafheituin, Berlin: "On the theory of Bessel's functions."

(13) A. Schoznfliess, Königsberg : "Report on the theory of point manifoldnesses."

(14) G. Hessenberg, Grunewald : "Involution for transfinite ordinal numbers."

(15) G. Liandsberg, Breslau : "On total curvature."

(16) K. RoHs, Leipzig: "Linear construction for cubic curves."

(17) Th. Schmid, Vienna : " Constructive treatment of the axis complex."

(18) H. Wiener, Darmstadt : "Models for twisted curves of the third order."

(19) C. JuEL, Copenhagen : "Non-analytic curves in three dimensions."

(20) R. MÜLLER, Brunswick : "Determination of the pole in motion of a plane system at branching points."

(21) C. RuNGE, Göttingen : "Graphical solutions of differential equations."

(22) R. Менмке, Stuttgart: "New mechanisms for solution of dynamical problems."

(23) A. Wagenmann, Stuttgart: "Mathematical theory of evolution."

1. Professor Blumenthal's paper was devoted to functions of infinite order. Reference was made to a new generalization of 
Picard's theorem by Rémoundos (Thèse, Paris, 1906). The speaker's results are to appear in a volume of the Collection de monographies, dirigée par Borel.

2. In comparison with the thorough investigations of the limits within which Fourier's series is valid, the treatment of Fourier's integral seems somewhat inadequate. In addition to integrability and conditions which are necessary for the series, the integral theorem requires also that the function satisfy certain complicated conditions at infinity. Professor Pringsheim undertakes to replace the latter by simple sufficient conditions by carrying over Dirichlet's condition (generalized by DuBoisReymond and C. Jordan) to intervals of the form $(A,+\infty)$, $(-A,-\infty)$. For this purpose he designates as quasi-monotone a function which is single-valued and finite in an interval $(A, B)$ and can be expressed as the sum of two finite, monotone parts. The condition is question may then be formulated: At infinity ( $i$. e., for $x>A$ and $x<-A$ where $A$ is an arbitrary positive quantity) $f(x)$ must converge towards zero monotone or quasi-monotone. The proof is to appear in the Annalen, also a more detailed account in the Jahresbericht.

3. Dr. Faber proved the following proposition: Denoting Legendre's polynomials by $P_{\nu}(x)$, the singular points $\beta$ of the function defined by the series $\sum_{v=0}^{\infty} a_{v} P_{\nu}(x)$ are connected with the singular points of the function $\sum_{\nu=0}^{\infty} a_{\nu} x^{\nu}$ by the relation $\beta=\frac{1}{2}\left(\alpha+\alpha^{-1}\right)$.

4. Dr. Perron generalized the following two propositions:

I. If a function $\sum a_{n} x^{n}$ admits on the circle of convergence only one singular point $\rho$, and if this is a rational pole, then $a_{n} / a_{n+1}$ approaches $\rho$ as a limit when $n$ becomes infinite (VivantiGutzmer, Theorie der eindeutigen analytischen Funktionen, page 397 ).

II. If there exists a limit $\rho=\lim _{n=\infty}\left(a_{n} / a_{n+1}\right)$, then $\rho$ is a singular point, although not necessarily a rational pole nor the only singular point on the circle of convergence. (Hadamard : La série de Taylor, page 25.)

Theorems I and II admit the following generalizations :

I $a$. If the singular points on the circle of convergence are all rational poles $\rho_{1}, \rho_{2}, \cdots, \rho_{k}$ of order $m_{1}, m_{2}, \cdots, m_{k}$ respectively and the greatest $m$ occurs $p$ times, then there exist $p$ numbers $\xi_{1}, \xi_{2}, \ldots, \xi_{p}$ such that 


$$
\lim \frac{a_{n}+\xi_{1} a_{n+1}+\cdots+\xi_{p} a_{n+p}}{\max \left|a_{n}, a_{n+1}, \cdots, a_{n+p}\right|}=0
$$

and the roots of the equation $\rho^{p}+\xi_{1} \rho^{p-1}+\cdots+\xi_{p}=0$ are precisely the $p$ poles with the highest degree of multiplicity.

I $b$. With the hypotheses of I $a$, if $\rho^{p-1}+\eta_{1} \rho^{p-2}+\cdots+$ $\eta_{p-1}$ is a divisor of $\rho^{p}+\xi_{1} \rho^{p-1}+\cdots+\xi_{p}$, the quotient

$$
\frac{\left|a_{n}+\eta_{1} a_{n+1}+\cdots+\eta_{p-1} a_{n+p-1}\right|}{\max \left|a_{n}, a_{n+1}, \cdots, a_{n+p-1}\right|}
$$

after a fixed value of $n$ remains greater than a certain positive number $\sigma$.

II $a$. If $p$ is the least number for which there exists a set of numbers $\xi_{1}, \xi_{2}, \cdots, \xi_{p}$ as in I $a$, and if, $\rho^{p-1}+\eta_{1} \rho^{p-2}+\cdots+$ $\eta_{p-1}$ being a divisor of $\rho^{p}+\xi_{1} \rho^{p-1}+\cdots+\xi_{p}$, the quotients

$$
\frac{\left|a_{n}+\eta_{1} a_{n+1}+\cdots+\eta_{p-1} a_{n+p-1}\right|}{\max \left|a_{n}, a_{n+1}, \cdots, a_{n+p-1}\right|}
$$

after a certain value of $n$ remain greater than a positive $\sigma$, then (1) the roots of the equation $\rho^{p}+\xi_{1} \rho^{p-1}+\cdots+\xi_{p}=0$ all have the same value ; $(2)$ this absolute value is equal to the radius of of convergence ; (3) the roots are singular points.

5. In connection with Weierstrass's lemma and the proofs by Poincaré and Cousin that a single-valued function meromorphic in the finite plane can be expressed as the quotient of two integral functions, Dr. Hartogs referred to a paper by Hahn in the Wiener Monatshefte in which the investigation of the zero-manifoldnesses of analytic functions of several variables is completely worked out and the problem of resolving integral functions into prime factors is solved. The investigation of those manifoldnesses which consist of the singular points of an analytic function is advanced on the one hand by ascertaining the general form of the region of convergence (Fabry, Comptes rendus, 1902 ; Faber, Mathematische Annalen, 61) and on the other by developing the analytic functions of $x$ and $y$ in series of the form $\sum f_{\nu}(x) \cdot y^{\nu}$ (Hartogs, Annalen, 62). Blumenthal has studied (Annalen, 57) those manifoldnesses which are common to the zero-manifoldnesses of several functions. 
6. Professor Stäckel showed that in many cases the CauchyWeierstrass inequalities can be advantageously replaced by inequalities similar in form but simpler in principle and for which it is not necessary to know the course of the function in the complex plane, but only the absolute values of the terms of the series. In this investigation of the power series for a single variable it is only necessary to assume that it converges for a value of the variable other than zero, but the corresponding assumption for power series of several variables is not sufficient. Here it is necessary to require unconditional convergence. The question whether the unconditional convergence can be deduced from less assumptions has been considered by F. Hartogs (Dissertation and Habilitationsschrift).

The speaker communicated a more general proposition, which he had proved conjointly with Hartogs, according to which it is sufficient to know that the simple series obtained by rearranging the multiple series can be made to converge uniformly in a region including the origin by a single rearrangement. Whether the conditions can be still further restricted remains undecided. A detailed account will appear in the Jahresbericht.

8. By aid of the theory of integral equations Dr. Hilb obtains extensions of Sturm's theorem and Klein's oscillation theorem for the equations

$$
\frac{d^{2} f}{d x^{2}}+k \phi(x) \cdot f(x)=0
$$

and

$$
\frac{\partial^{2} f}{\partial x^{2}}+\frac{\partial^{2} f}{\partial y^{2}}+k\left[\phi(x)-\phi_{1}(y)\right] f\left(x_{1} y\right)=0
$$

respectively, if in (1) within a segment of the $x$-axis $\phi(x)$ is sometimes positive, sometimes negative, and if in $(2) \phi(x)$ is now greater, now less than $\phi_{1}(y)$. Sturm's theorem then becomes : To every oscillation number $m$ correspond a positive and a negative value for which a particular function exists that vanishes $m$ times within the segment. Klein's oscillation theorem becomes : To every oscillation pair $m, n$ correspond two values (positive and negative) for which a solution of (2) exists that can be expressed in the form $h(x) \cdot g(y)$, where $h(x)$ vanishes $m$ times and $g(y) n$ times within the segments. By an extension of the method which Dr. Hilb has developed in an article “ Über die Reihentwickelungen der Potentialtheorie," to appear 
in the next number of the Annalen, it follows that all the particular functions of (2) which vanish on the boundary of the rectangle formed by the two segments are identical with produsts $h(x) \cdot g(y)$. Hence we obtain the development in terms of such products.

9. Following some investigations of Borel, Professor Krause showed how continuous functions can be developed in uniformly convergent series of rational integral functions. He connected this with the known expressions for continuous functions in convergent series of rational integral functions and showed that under certain conditions uniformly convergent series can be assigned to the given functions.

10. Dr. Koebe proved the proposition that every triply connected plane region can be mapped conformally on a singleleaved region bounded by three circles which do not cut nor touch one another. He also gave a method of solving the corresponding problem for all $n$-ply connected single-leaved regions which are symmetric with respect to the real axis and have the property that every boundary cuts the real axis. The paper will appear in the Jahresbericht.

11. Professor Meyer extends Euclid's algorithm to rational integral functions $r_{0}(\lambda), r_{1}(\lambda), \cdots, r_{\kappa-1}(\lambda)$. The simultaneous vanishing of the $\kappa-1$ last remainders is the necessary and sufficient condition for the existence of a variable common factor of the $\kappa$ given polynomials, and the last remainder which does not vanish gives their greatest common divisor. Every one of these remainders can be expressed linearly in the given $r$ 's,

$$
R_{i}=A_{0}^{(i)} r_{0}+A_{1}^{(i)} r_{1}+\cdots+A_{\kappa-1}^{(i)} r_{\kappa-1}(i=1,2, \cdots \kappa-1) .
$$

An integral function of the coefficients of the $r$ 's which vanishes whenever two or more $r$ 's have a common root is called a partial resultant. Every partial resultant may be written

$$
R=A_{0} r_{0}+A_{1} r_{1}+\cdots+A_{\kappa-1} r_{\kappa-1} .
$$

If to the $\kappa$ identities I and II we add the relation

$$
0=B_{0} r_{0}+B_{1} r_{1}+\cdots+B_{k-1} r_{k-1},
$$

and eliminate the $r$ 's, it follows that every partial resultant of 
the given polynomials, multiplied by a suitable $A$, can be expressed linearly in terms of the last remainders $R_{1}, R_{2}, \cdots, R_{\kappa-1}$.

$$
A R=A_{1} R_{1}+A_{2} R_{2}+\cdots+A_{\kappa-1} R_{\kappa-1} .
$$

12. In a paper presented to the Berlin Mathematical Society, Professor Schaf heitlin proved that the first root of the Bessel's function of the second kind $y_{n}(x)$ is greater than $n$ and that these roots obey the law obtained by Schläfli for Bessel's functions of the first kind $J_{n}(x)$, namely, that each root, regarded as a function of $n$, increases with $n$. In the present paper it is proved that Schläfli's proposition is also true for all the roots of $d J_{n} / d x$ and for those of $d y_{n} / d x$ which are greater than the first root of $y_{n}$. For small values of $n$ these are all the roots of $d y_{n} / d x$. It appears that the first root of $d^{2} y_{n} / d x^{2}$, for small values of $n$, follows that of $y_{n}$ but for $n>7.5$, two roots of $d^{2} y_{n} / d x^{2}$ precede those of $y_{n}$. Thus the curve for $y_{n}(x)$, after cutting the $x$-axis to the right of $x=n$, is convex towards it, for small values of $n$. But for larger values of $n$ it has two turning points before cutting the $x$-axis.

13. Professor Schoenfliess's report will appear in full in the Jahresbericht.

14. Professor Hessenberg defines the covering $x$ of a finite part $S_{1}$ of a class $S$ with elements of a class $R$ as a finite covering of $S$ with $R$, and denotes by $(R / S)$ the class of all these finite coverings. If $S$ and $R$ are simply ordered, $(R / S)$ can be simply ordered and its ordinal type $(\rho / \sigma)$ possesses the properties of a power of $1+\rho$ (Hausdorff, Leipziger Berichte, volume 26). If further $R$ and $S$ are well ordered, then by the process referred to $(R / S)$ is also well ordered and $(\rho / \sigma)$ is Cantor's power $(1+\rho)^{\sigma}$ which possess the defining properties

$$
a^{1}=a, \quad a^{\beta+1}=a^{\beta} a, \quad \lim _{\beta} a^{\beta}=a^{\lim \beta} .
$$

The author obtains a new proof of this last proposition.

15. Professor Landsberg pointed out that the theorems of Gauss and O. Bonnet on total curvature of surfaces can be, to a certain extent, carried over to the general problem of calculus of variations. For this purpose he classifies the problems which lead to the integral

$$
s=\int \phi\left(x, y, x^{\prime}, y^{\prime}\right) d t
$$


according to the degree $m$ to which $x^{\prime}$ and $y^{\prime}$ enter into the equation of condition

$$
T=\phi_{x^{\prime}} \cdot \delta x+\phi_{y^{\prime}} \cdot \delta y=0
$$

for the transformed position. If $m=1$ and if the relation for the new position is an involution,

$$
\phi=\sqrt{E x^{\prime 2}+2 F x^{\prime} y^{\prime}+G y^{\prime 2}}
$$

Then, depending on the sign of the discriminant, we obtain Gauss's form for the element of length or one in which the minimal curves are real. In both cases the concepts of geodetic curvature of a curve at a point, curvature of the field at a point, total curvature of a region can be so stated that an extension of the above two-dimensional domain is unnecessary. It follows that the change in the length of an arc on the surface due to a uniform transverse displacement of its points is equal to the mean value of the surface curvature along this curve; if the curve is contracted to an element of arc, the change is equal to the surface curvature itself. The Gauss-Bonnet theorem on curvatura integra of a portion of a surface has also its exact analogon in the case where the minimal curves are real.

17. If from every point $P$ of a surface of the second order the normals $p$ to the polar plane $\pi$ are drawn, a one-to-one relation is established between the axis complex $p$ and both the space $P$ and the space $\pi$. From these relations Professor Schmid obtains the focal properties, constructions for the normals and for the principal centers of curvature, by projecting $P$ and $p$ from the center $O$, and the other vertices of the principal polar tetraedron on the corresponding opposite faces. The substance of the paper may be found in the Sitzungsberichte of the Vienna Academy for 1906 (volume 115, part II $a$ ).

18. Professor Wiener exhibited two models for space curves of the third order, regarding such a curve 1) as the common section of two cones with a common generatrix ; 2) as enveloped by two planes which touch two conics with a common tangent. In both cases we meet with the osculating tetraedron determined by two points of the curve with their tangents and osculating planes. In 1) the vertices of the cones are the two 
points of the curve; in 2) the planes of the conics are the osculating planes. Each model shows the curve by aid of its tangents in strings and the osculating tetraedron by wires. They complete the set of which twelve have been exhibited and will appear with these in the Teubner series.

19. Non-analytic curves can be classified according to their order and the class and number of singular points investigated by purely geometric methods. Professor Juel treated in this way curves of the fourth order obtained as intersections of two (non-analytic) cones of the second order and a curve of the $n$th degree lying on a hyperboloid and cutting every generating line of one set $n-1$ times. For both classes the same characteristic numbers were obtained as in the algebraic cases. The question was also considered whether such curves have any real existence as non-analytic.

20. In the motion of a similarly deformable plane system the usual construction for the pole - depending on the tangents to the paths at three arbitrary points of the system * — fails when these tangents intersect on the circle through the three points. Professor Müller considered this case in the light of his results in a paper "Ueber die Krümmungsmittelpunkte der Bahnkurven in ebenen ähnlichveränderlichen Systemen" (Zeitschrift für Mathematik und Physik, volume 36, page 129) and showed how to determine the pole and that the system admits two initial motions from the position in question.

21. Professor Runge adapted Picard's method of successive approximations to the graphic solution of differential equations of the first order. The curve is first replaced by an approximating broken line so drawn that its integral wherever it cuts the curve in the direction of the $x$-axis agrees with the curve integral. The curve is here replaced by arcs of parabolas with axes parallel to the axes of $x$ or $y$. In this way it is possible to determine the positions of the vertical parts of the broken line so that the areas above and below the curve shall be equal. On the surface $z=f(x, y)=d y / d x$, every curve $f(x, y)=$ const. determines a direction. A rough approximation to the required curve being drawn, a piece of it $A B$ is taken small enough so that the above directions for $A B$ do not include too large

\footnotetext{
* Burmester, Kinematik I, p. 867.
} 
angles. This approximation is then made closer by using the values of $f$ at points where $A B$ cuts the curve $f(x, y)=$ const. If the second approximation is not close enough, the process is repeated.

23. Herr Wagenmann correlates successive steps in the theory of evolution with series $-\infty, \cdots-2,-1,0,1,2, \cdots, \infty$ along three coördinate axes developing successively the ideas of motion, mass, the nebular hypothesis and evolution of living organisms and of civilization. He finds that his method leads to a monistic philosophy - in fact to a pan-monism.

\section{Göttingan,}
A. B. Frizeil.

November, 1906.

\section{A NEW APPROXIMATE CONSTRUCTION FOR $\pi$.}

BY MR. GEORGE PEIRCE.

Given a circle with radius $r$ and center at $O$; to find an approximate construction for $\pi r$.

Draw the diameter $A O B$ and the tangent $B C$ at right angles to it. Describe the arc $O D C$ with radius $r$ and center at $B$.

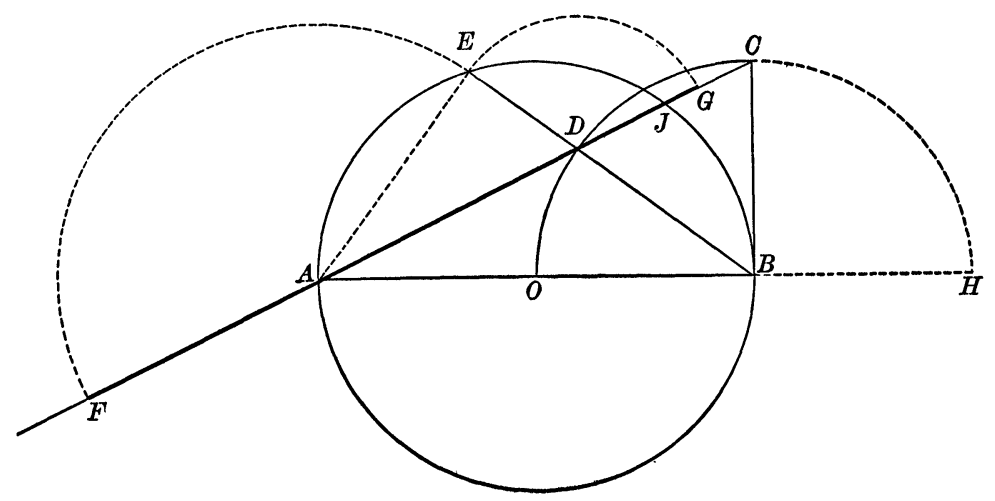

Draw the line $A C$ cutting the arcs $O D C$ and $A B$ at $D$ and $J$; also draw the line $B D E$ through $B$ and $D$ cutting the given circle at $E$. Then $A D+3 D E=\pi r$ approximately. 